\title{
Cerebral Perfusion After Repair of Congenital Diaphragmatic Hernia with Common Carotid Artery Occlusion After ECMO Therapy
}

\author{
CLAUDIA HENZLER ${ }^{1}$, FRANK G. ZÖLLNER ${ }^{2}$, MEIKE WEIS ${ }^{1}$, FABIAN ZIMMER ${ }^{2}$, \\ STEFAN O. SCHOENBERG ${ }^{1}$, KATRIN ZAHN ${ }^{3}$, THOMAS SCHAIBLE ${ }^{4}$ and K. WOLFGANG NEFF ${ }^{1}$ \\ ${ }^{1}$ Institute of Clinical Radiology and Nuclear Medicine, Departments of ${ }^{3}$ Pediatric Surgery, and \\ ${ }^{4}$ Neonatology, University Medical Center Mannheim, University of Heidelberg, Mannheim, Germany; \\ ${ }^{2}$ Computer Assisted Clinical Medicine, Medical Faculty Mannheim, University of Heidelberg, Mannheim, Germany
}

\begin{abstract}
Aim: To prospectively evaluate cerebral perfusion after repair of congenital diaphragmatic hernia $(C D H)$ and right-common-carotid-artery (rCCA) occlusion after extracorporeal membrane oxygenation (ECMO) therapy. Patients and Methods: A total of 29 2-year-old-children with a history of $\mathrm{CDH}$ repair underwent cerebral magnetic resonance perfusion imaging. In 14 patients, the rCCA was occluded after ECMO therapy. Fifteen patients with $C D H$ without ECMO served as controls. Regional cerebral-bloodflow (rCBF) was measured cortically and subcortically in both hemispheres and compared intra-individually and between both groups. Results: Patients with rCCA-occlusion showed intra-individual side differences between hemispheres, with significantly lower subcortical perfusion of the right hemisphere and reduced cortical perfusion. In one-third of patients with rCCA-occlusion, $r C B F$ of the right-hemisphere was reduced by more than $20 \%$ when compared to the left hemisphere. Despite intra-individual side differences, mean $\mathrm{rCBF}$ in patients with $\mathrm{rCCA}$ occlusion was not reduced compared to controls. Conclusion: Beside intra-individual side differences, overall right hemisphere perfusion is sufficient after rCCA-occlusion due to collateral blood supply.
\end{abstract}

This article is freely accessible online.

Correspondence to: Claudia Henzler, MD, Institute of Clinical Radiology and Nuclear Medicine, University Medical Center Mannheim, Medical Faculty Mannheim, Heidelberg University, Theodor-Kutzer-Ufer 1-3, 68167 Mannheim, Germany. Tel: +49 6213832067, Fax: +49 6213831910, e-mail: claudia.henzler@ medma.uni-heidelberg.de

Key Words: Congenital diaphragmatic hernia, pulsed arterial spin labeling, extracorporeal membrane oxygenation therapy, common carotid artery occlusion, cerebral perfusion.
Congenital diaphragmatic hernia (CDH) occurs in approximately one in 3,000-4,000 live births (1). A defect of the diaphragm leads to herniation of abdominal organs into the thoracic cavity. Lung hypoplasia and secondary pulmonary hypertension are the major causes of death (2). The implementation of new therapeutic strategies has improved the survival rate even in those patients with severe $\mathrm{CDH}$ (3). If conservative treatment in newborns with $\mathrm{CDH}$ fails, extracorporeal membrane oxygenation (ECMO) therapy is an important alternative for accomplishing sufficient oxygenation of the newborns. The Congenital Diaphragmatic Hernia Study Group showed an improved survival rate in patients with $\mathrm{CDH}$ after ECMO therapy, in whom, without ECMO, mortality was predicted to be higher than $80 \%$ (4). As a consequence, morbidity plays an increasing role in patients with $\mathrm{CDH}$. Not only pulmonary problems but also neurological disorders have been identified as significant morbidities among $\mathrm{CDH}$ survivors $(5,6)$. In a study by McGahren et al., 8/12 (67\%) ECMO survivors showed delayed neurological development compared to only $5 / 21(24 \%)$ of the non-ECMO-treated survivors.

In $\mathrm{CDH}$, veno-arterial ECMO is usually performed. Vascular access is usually achieved by insertion of the ECMO cannulas through the right common carotid artery (rCCA) and the right internal jugular vein. At the time of decannulation, ligation or surgical reconstruction of the rCCA can be performed but secondary occlusion of the surgical reconstructed rCCA can occur (7). Büsing et al. investigated 2-year-old children with history of $\mathrm{CDH}$ and reconstruction of the rCCA after ECMO therapy. Their study showed that in $72 \%$ of the patients, the rCCA was found to be occluded or highly stenotic using contrast-enhanced magnetic resonance angiography (7). Using Doppler ultrasound in 5-year-old patients, Desai et al. reported that most of the reconstructed rCCAs after ECMO therapy were patent and in only $24 \%$ of the patients there was significant 
stenosis or occlusion of the vessel (8). It remains unclear whether an occlusion of the rCCA leads to reduced perfusion of the right cerebral hemisphere.

Pulsed arterial spin labeling (pASL) magnetic resonance imaging (MRI) is a non-invasive method for assessing cerebral perfusion. Using magnetically labeled water in arterial blood as an endogenous contrast material, there is no need for administration of contrast agent or radioactive tracers $(9,10)$. Therefore, pASL is a well-suited perfusion imaging technique for pediatric patients (11).

The purpose of this study was to prospectively evaluate cerebral perfusion in 2-year-old children after $\mathrm{CDH}$ repair using quantitative pASL MRI at 3.0 T and to assess whether occlusion of the rCCA after neonatal ECMO therapy leads to impaired cerebral perfusion.

\section{Patients and Methods}

Patients. A total 29 2-year old patients (mean age 2.1 \pm 0.2 years, 20 male) after neonatal $\mathrm{CDH}$ repair were included in this study. All patients were prospectively investigated at our Department according to our long-term follow-up program, which includes an MRI examination at the age of 2 years. The study was approved by our Institutional Review Board (2013-818R-MA) and complies with the Declaration of Helsinki. Written informed parental consent was obtained for all children enrolled in this study.

In 14 patients (10 male, 4 female), the rCCA was occluded at the MRI examination at the age of 2 years even though carotid artery reconstruction after neonatal ECMO therapy was conducted. The remaining 15 patients ( 11 male, 4 female) with history of $\mathrm{CDH}$ but without ECMO therapy and normal cerebral blood supply served as a control group. In patients with occlusion of the rCCA, the mean age at MRI examination was $2.1 \pm 0.2$ years and $2.0 \pm 0.1$ years in the control group without ECMO therapy.

Neonatal ECMO therapy. When neonatal ECMO therapy was required, informed parental consent was obtained. ECMO therapy was administered according to previously published guidelines (12, 13). In all patients, veno-arterial ECMO therapy was performed via cannulation of the rCCA and the right internal jugular vein.

Carotid artery reconstruction. At the time of decannulation, the right internal jugular vein was ligated and carotid artery reconstruction was carried out. For surgical reconstruction, the segment of cannula insertion was excised and an end-to-end anastomosis with interrupted 7.0 Prolene sutures was performed (Figure 1). After surgery, continuous heparin infusion $(200 \mathrm{IU} / \mathrm{kg}$ ) was given for the next 3 days followed by $100 \mathrm{IU} / \mathrm{kg}$ daily for another 4 weeks.

MRI. At the age of 2 years, the routine follow-up program in children after $\mathrm{CDH}$ repair at our Institution included MRI of the brain and the chest. All children underwent sedation for the MRI examination by intravenous administration of propofol and continuous intensive care monitoring was performed by an anesthesiologist. Images were acquired during spontaneous free breathing of the sedated children. All patients were examined using a 3.0 T MRI system (Magnetom TimTrio; Siemens Healthcare Sector, Erlangen, Germany). A combination of spine array, head and neck coil and body phased-array coil was used. Brain imaging in the follow-up program included T1- and T2-weighted images, as well as diffusion-weighted imaging (DWI), diffusion tensor imaging, fluid attenuated inversion recovery (FLAIR) and pASL perfusion imaging.

Using T1- and T2-weighted imaging, DWI with apparent diffusion coefficient calculation and a FLAIR sequence, all patients were evaluated for focal cerebral lesions by two radiologists in a consensus reading.

Cerebral perfusion was measured in all patients using a commercially available pASL sequence (Siemens Healthcare Sector). For pASL, a multistep spin-echo EPI (echo planar imaging) sequence with PICORE (proximal inversion with a control for offresonance effects) labeling scheme and Q2TIPS (QUIPSS II, Quantitative imaging of perfusion using a single subtraction, second version) (14). Scan parameters of the pASL sequence were as follows: $100 \mathrm{~mm}$ labeling region with $25 \mathrm{~mm}$ gap between the labeling region and image section, $\mathrm{TI}_{1}$ (inversion time 1) $=700 \mathrm{~ms}$, $\mathrm{TI}_{1} \mathrm{~s}$ (saturation stop time) $=1600 \mathrm{~ms}, \mathrm{TI}_{2}$ (inversion time 2$)=1800$ $\mathrm{ms}, \mathrm{TR} / \mathrm{TE}$ (repetition time/echo time) $=2474.6 / 21 \mathrm{~ms}$, FOV (field of view) $210 \times 210 \mathrm{~mm}^{2}$, voxel size $2.2 \times 2.2 \times 8.0 \mathrm{~mm}^{3}$. Tag and control images were averaged over 100 images (50 control and label image pairs) resulting in a total scan time of $4 \mathrm{~min}$. Based on the pASL sequence, two perfusion images, a perfusion-weighted image (subtraction image of label and control pairs) and a regional cerebral blood flow (rCBF) map (scaled to units of $\mathrm{ml} / 100 \mathrm{~g} / \mathrm{min}$ ) were automatically generated.

To evaluate the vascular status of the patients, the follow-up MRI examination at the age of 2 years also included an intracranial 3D time-of-flight angiography as well as an intracranial, cervical and pulmonary contrast-enhanced time-resolved angiography with stochastic trajectory (TWIST) angiography (Figure 2) (15). A solution consisting of $0.05 \mathrm{mmol}$ contrast agent (Dotarem;Guerbet, France) per kilogram body weight and the same amount of sodium chloride followed by a bolus of $10 \mathrm{ml}$ of sodium chloride were administered at a flow of $1 \mathrm{ml} / \mathrm{s}$.

Data analysis. In order to assess differences in cerebral perfusion between hemispheres, one cortical (mainly containing cortical gray matter) and one subcortical (containing subcortical gray and white matter) region of interest (ROI) was manually drawn by one radiologist in each hemisphere in the territory of the middle cerebral artery at the level of the lateral ventricles based on pASL images superimposed with $\mathrm{T} 2$-weighted morphological reference images (Figure 3) using the fusion tool on an off-line workstation (Aycan Osirix pro, version 5.0.2; Aycan, Würzburg, Germany). The radiologist was blinded to patient history. In total, four ROIs were evaluated per child. ROIs did not include larger arteries and veins.

Statistical analysis. Quantitative parameters are reported as mean values \pm standard deviation. Paired $t$-test was applied to compare the mean $\mathrm{rCBF}$ of the right and left hemispheres in both patient groups for cortical and subcortical regions. In order to obtain a relative parameter, a ratio of the $\mathrm{rCBF}$ of the right and left hemispheres was additionally calculated for each patient $\left(\mathrm{rCBF}_{\text {right }} / \mathrm{rCBF}_{\text {left }}\right)$. To compare the $\mathrm{rCBF}_{\text {right }} / \mathrm{rCBF}_{\text {left }}$ ratio between groups with and without rCCA occlusion, Student's two-sample $t$-test was used. A $p$-value of less than 0.05 was considered statistically significant. Statistical analysis and graphical presentation of data were performed with Prism, version 5.0a (GraphPad Software, La Jolla, CA, USA). 


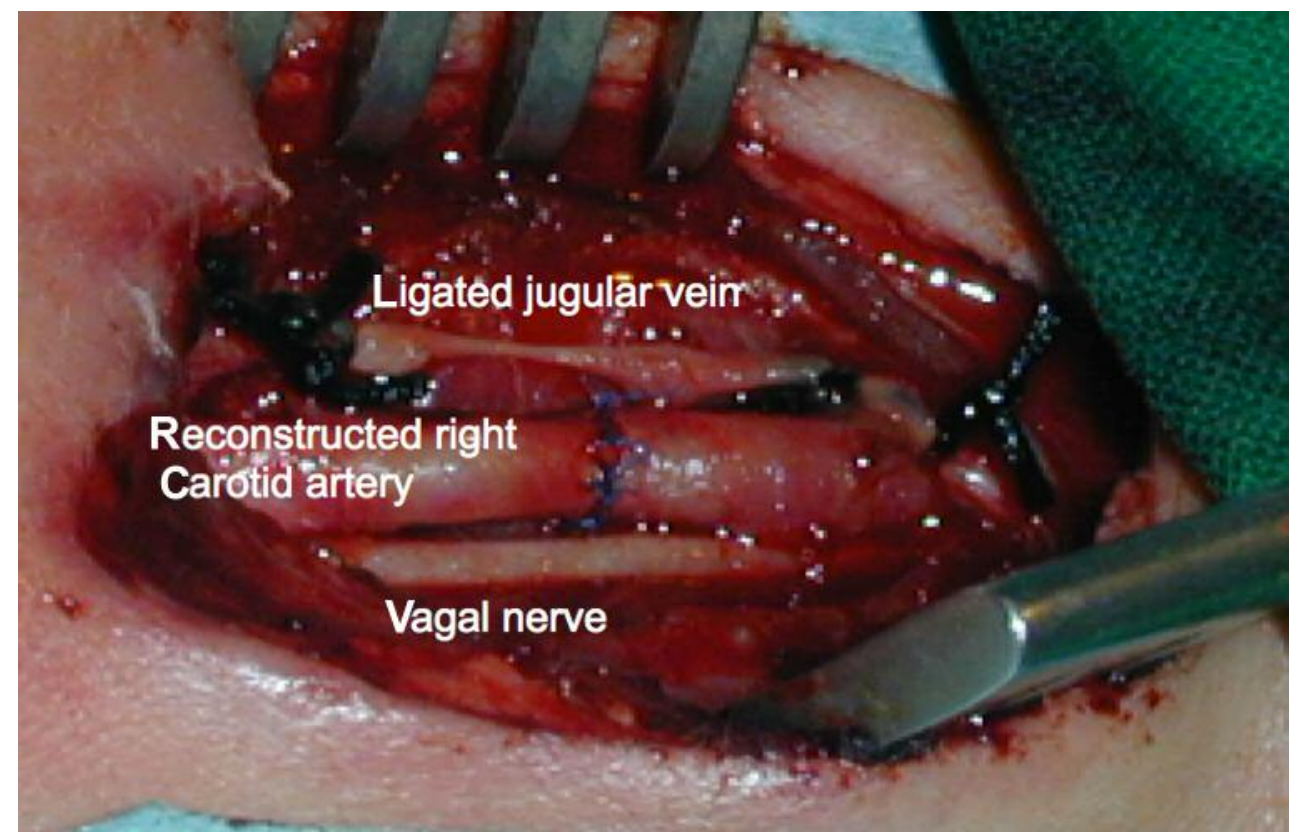

Figure 1. Intraoperative view of reconstructed right common carotid artery after extracorporeal membrane oxygenation decannulation in a newborn with congenital diaphragmatic hernia.
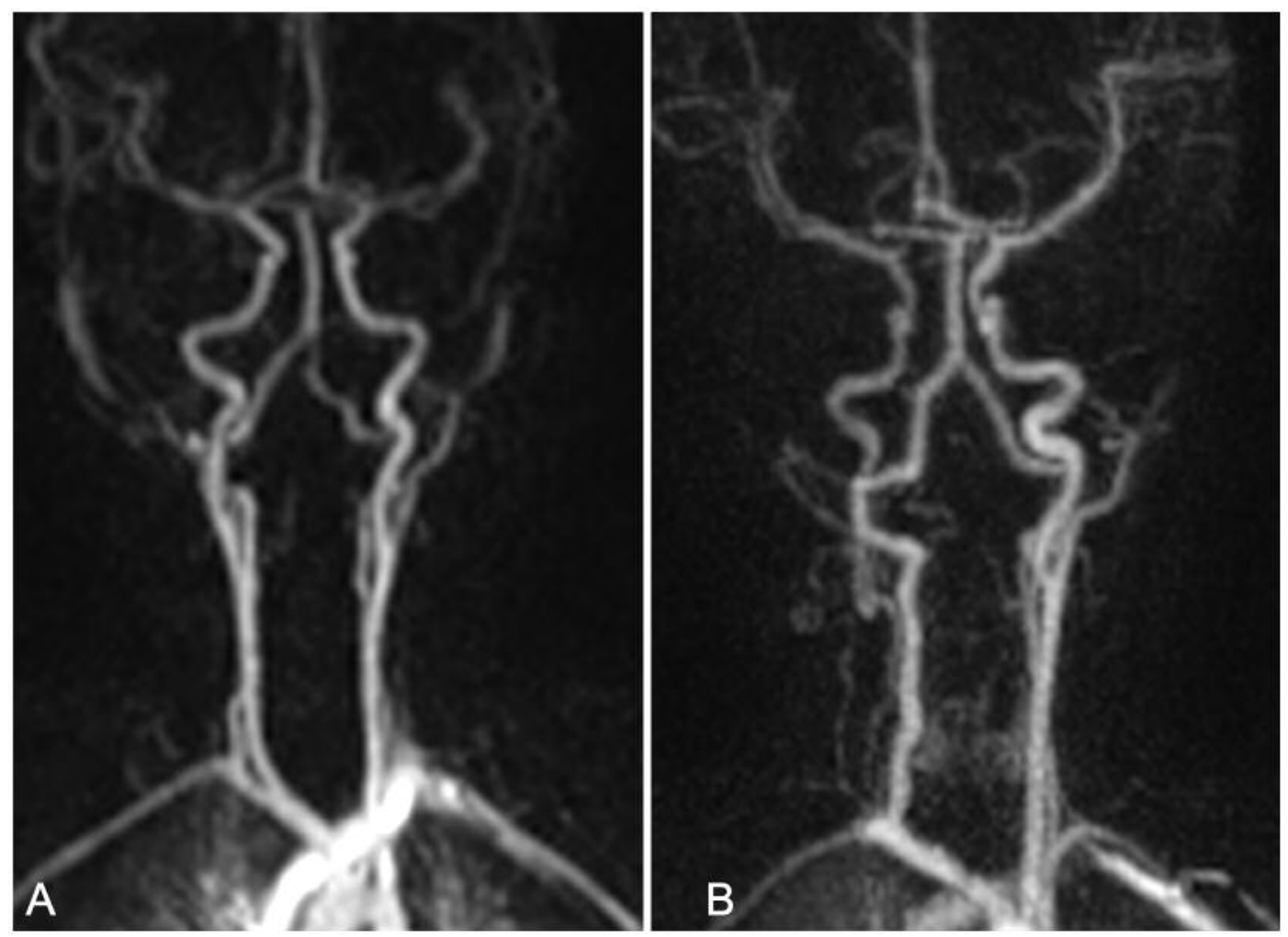

Figure 2. Time-resolved angiography with stochastic trajectory (TWIST) angiography in a 2-year-old patient without extracorporeal membrane oxygenation therapy and normal cerebral/cervical blood supply $(A)$ and in a 2-year-old patient with occlusion of the right common carotid artery and cervical collateral blood supply via the thyreocervical trunk filling the right internal carotid artery (B). In comparison to the left internal carotid artery, the diameter of the right internal carotid artery is reduced $(B)$. 


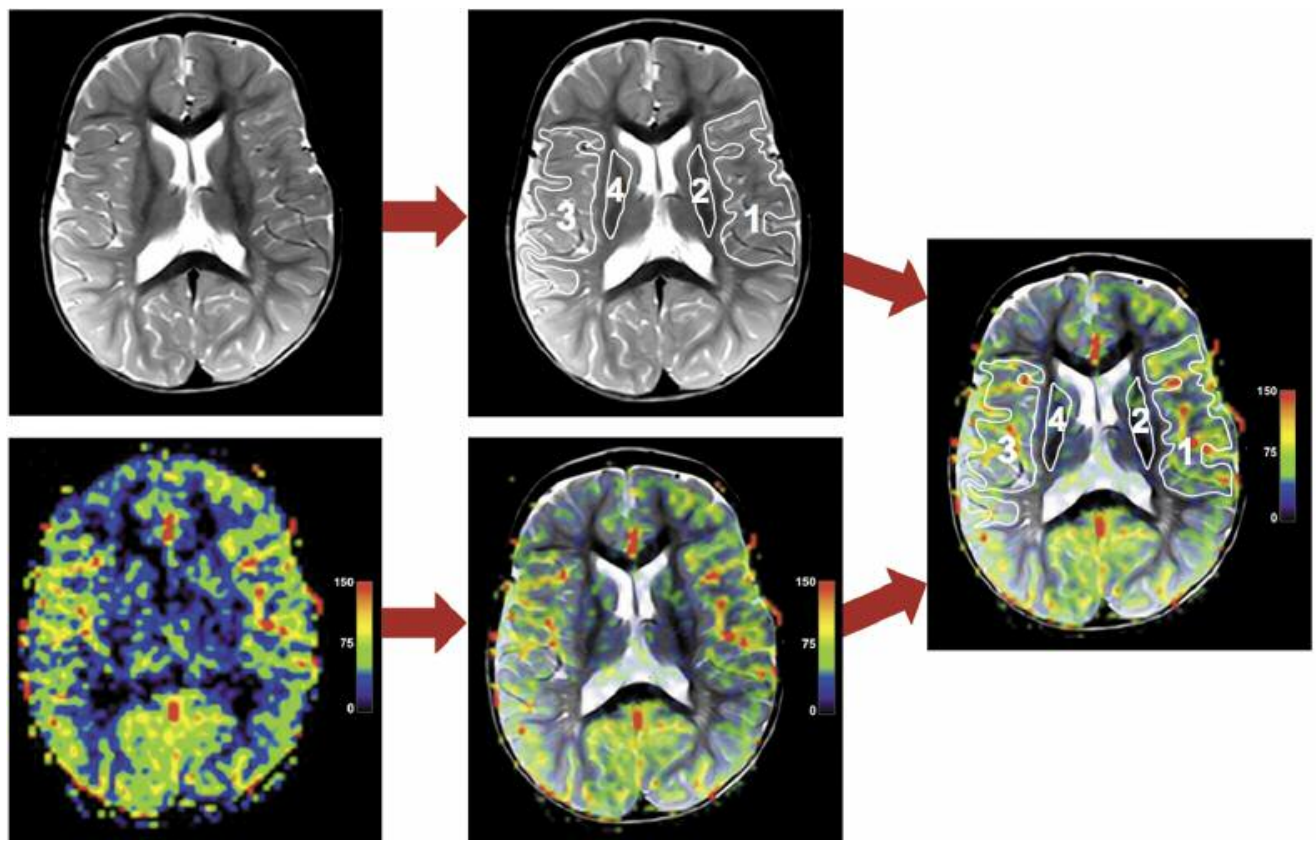

Figure 3. Pulsed arterial spin labeling image superimposed with T2-weighted morphological image. Cortical (1 and 3) and subcortical (2 and 4) regions of interest.

\section{Results}

pASL based regional cerebral blood flow. In patients with rCCA occlusion after neonatal ECMO therapy, the mean subcortical $\mathrm{rCBF}$ was $68.8 \pm 23.6 \mathrm{ml} / 100 \mathrm{~g} / \mathrm{min}$ for the right hemisphere and $79.1 \pm 30.5 \mathrm{ml} / 100 \mathrm{~g} / \mathrm{min}$ for the contralateral left hemisphere. Subcortical perfusion of the right hemisphere in children with rCCA occlusion was significantly lower when compared to that of the left hemisphere $(p=0.011)$.

Cortical perfusion showed a trend towards a reduced perfusion of the right hemisphere with a $\mathrm{rCBF}$ of $65.9 \pm 24.5$ $\mathrm{ml} / 100 \mathrm{~g} / \mathrm{min}$ versus $72.2 \pm 29.0 \mathrm{ml} / 100 \mathrm{~g} / \mathrm{min}(p=0.138)$. No patient of our study cohort showed intracerebral ischemic lesions, residual after intracranial hemorrhage, or other focal cerebral lesions.

The control group without ECMO therapy and normal blood flow of the rCCA displayed nearly identical subcortical cerebral perfusion of both hemispheres, with a mean subcortical $\mathrm{rCBF}$ of $54.8 \pm 26.9 \mathrm{ml} / 100 \mathrm{~g} / \mathrm{min}$ for the right hemisphere and $56.2 \pm 24.2 \mathrm{ml} / 100 \mathrm{~g} / \mathrm{min}$ for the left hemisphere $(p=0.340)$. Mean cortical rCBF was also nearly identical between the right and left hemisphere $(61.3 \pm 25.3 \mathrm{ml} / 100 \mathrm{~g} / \mathrm{min} v s .61 .6 \pm 25.4$ $\mathrm{ml} / 100 \mathrm{~g} / \mathrm{min}, p=0.780$; Figures 4 and 5).

Comparing the $\mathrm{rCBF}$ of the same hemisphere between patients with rCCA occlusion after ECMO therapy and patients of the control group, the mean $\mathrm{rCBF}$ in patients with

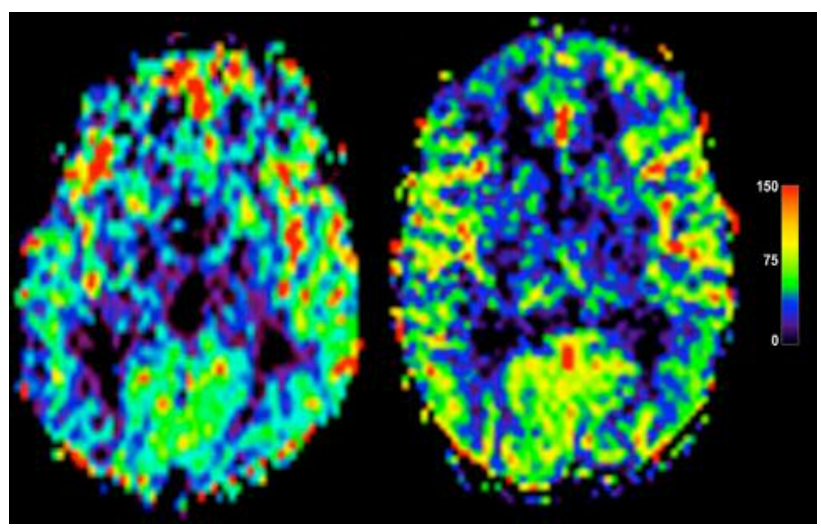

Figure 4. Pulsed arterial spin labeling images of a patient with occlusion of the right common carotid artery after extracorporeal membrane oxygenation therapy and lower perfusion of the right hemisphere (left) compared to a patient of the control group with normal cerebral blood supply (right).

rCCA occlusion was always higher. The mean subcortical rCBF of the left hemisphere in patients with rCCA occlusion was significantly higher when compared with that of the control group $(p=0.034)$. Regarding the subcortical rCBF of the right hemisphere, there was no statistically significant difference between patient groups $(p=0.148)$. There was also no significant difference between study groups with regard 

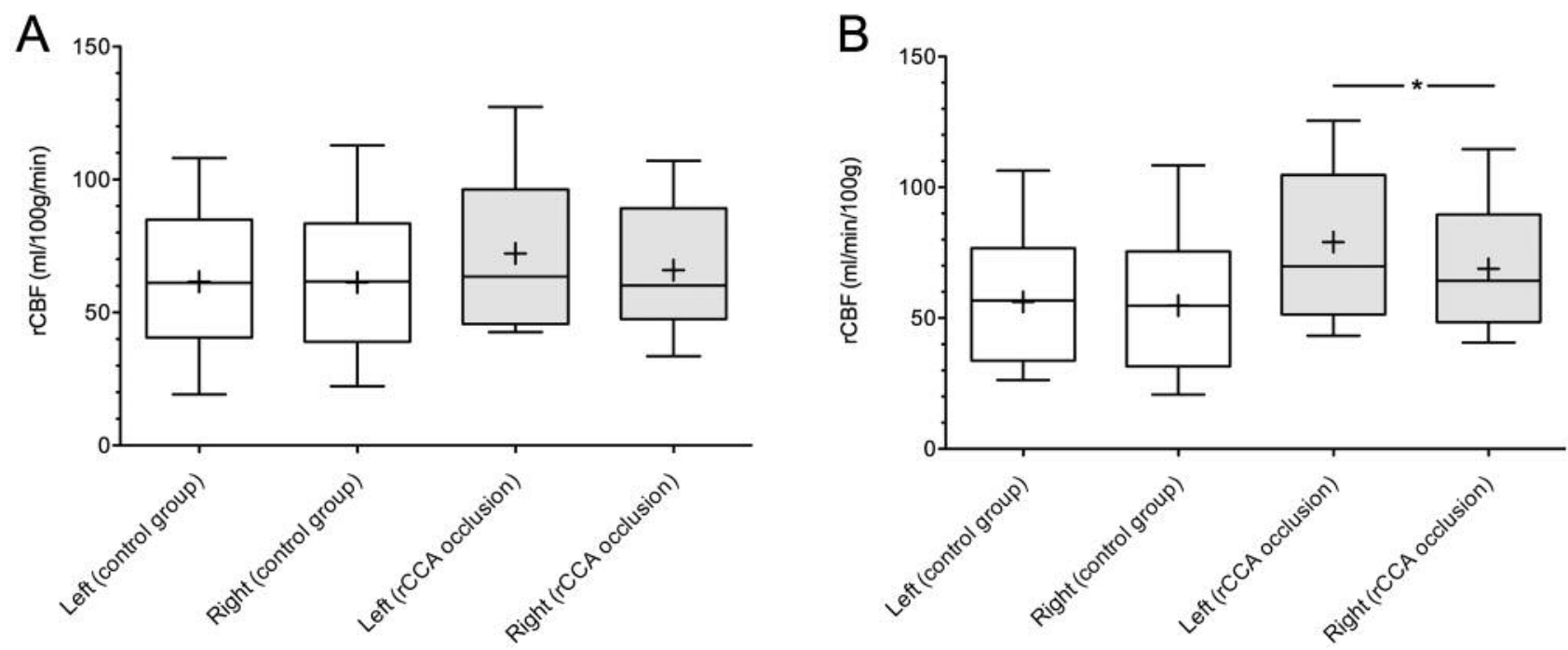

Figure 5. Boxplots of cortical (A) and subcortical $(B)$ regional cerebral blood flow ( $r C B F)$ of both hemispheres in the non-extracorporeal membrane oxygenation (ECMO) group (control group) and ECMO group with right common carotid artery (rCCA) occlusion. +: mean value, *denotes significant difference.

to the mean cortical rCBF of the left hemisphere $(p=0.302)$ or the right hemisphere $(p=0.623)$.

Ratio $r C B F_{\text {right }} / r C B F_{\text {left }}$. In order to obtain a relative parameter, the ratio of the $\mathrm{rCBF}$ of the right and left hemispheres $\left(\mathrm{rCBF}_{\text {right }} / \mathrm{rCBF}_{\text {left }}\right)$ was calculated for each patient (Table I). The mean ratio of the cortical $\mathrm{rCBF}$ of the right and left hemisphere $\left(\mathrm{rCBF}_{\text {right }} / \mathrm{rCBF}_{\text {left }}\right)$ was $1.00 \pm 0.08$ in the non-ECMO group and $0.94 \pm 0.20$ in children with rCCA occlusion. For the subcortical ROIs, the mean $\mathrm{rCBF}_{\text {right }} / \mathrm{rCBF}_{\text {left }}$ ratio was $0.95 \pm 0.11$ in the control group and $0.89 \pm 0.14$ in the group with rCCA occlusion. No statistically significant differences in the $\mathrm{rCBF}_{\text {right }} / \mathrm{rCBF}_{\text {left }}$ ratio were demonstrated between patients with and without rCCA occlusion, for both cortical $(p=0.260)$ and subcortical $(p=0.221)$ ROIs.

In four patients with occlusion of the rCCA (29\%), the rCBF of the right hemisphere was reduced by more than $20 \%$ when compared with the contralateral hemisphere (Table I). In a subgroup analysis of these four patients, the mean cortical $\mathrm{rCBF}$ of the right hemisphere was $55.7 \pm 25.3 \mathrm{ml} / 100 \mathrm{~g} / \mathrm{min}$. There was no significant difference between these four patients and the control group regarding mean cortical $\mathrm{rCBF}$ of the right hemisphere $(55.7 \pm 25.3 \mathrm{ml} / 100 \mathrm{~g} / \mathrm{min} v s$. $61.3 \pm 25.3 \mathrm{ml} / 100 \mathrm{~g} / \mathrm{min}, p=0.702)$. The mean subcortical $\mathrm{rCBF}$ of the right hemisphere of the four patients with an intra-individual side difference of more than $20 \%$ between hemispheres was even higher when compared with the control group $(75.3 \pm 22.6 \mathrm{ml} / 100 \mathrm{~g} / \mathrm{min} v s .54 .8 \pm 26.9 \mathrm{ml} / 100 \mathrm{~g} / \mathrm{min})$ but without statistically significant difference $(p=0.183)$.
Table I. Ratio of the cortical and subcortical regional cerebral blood flow $(r C B F)$ of the right and left hemisphere $\left(r C B F_{\text {right }} / r C B F_{\text {left }}\right)$ in individual congenital diaphragmatic hernia $(C D H)$ children without extracorporeal membrane oxygenation (ECMO) therapy (controls) and in children with right-common-carotid-artery (rCCA) occlusion after ECMO therapy.

\begin{tabular}{|c|c|c|c|}
\hline \multicolumn{2}{|c|}{ Cortex } & \multicolumn{2}{|c|}{ Subcortex } \\
\hline Controls $(n=15)$ & $\begin{array}{l}\text { rCCA occlusion } \\
\quad(n=14)\end{array}$ & $\begin{array}{c}\text { Controls } \\
(n=15)\end{array}$ & $\begin{array}{l}\text { rCCA occlusion } \\
(\mathrm{n}=14)\end{array}$ \\
\hline 0.98 & 0.85 & 0.95 & 0.98 \\
\hline 0.99 & 0.70 & 1.05 & 0.70 \\
\hline 0.98 & 0.86 & 1.02 & 1.03 \\
\hline 0.95 & 0.79 & 0.81 & 0.94 \\
\hline 0.89 & 1.22 & 0.96 & 0.82 \\
\hline 1.04 & 1.27 & 1.11 & 1.16 \\
\hline 1.11 & 0.80 & 0.82 & 0.90 \\
\hline 0.97 & 0.89 & 1.14 & 0.92 \\
\hline 1.02 & 1.11 & 1.02 & 0.80 \\
\hline 0.95 & 0.67 & 0.95 & 0.71 \\
\hline 1.16 & 0.77 & 0.88 & 0.77 \\
\hline 0.99 & 0.99 & 0.79 & 1.01 \\
\hline 0.95 & 1.10 & 0.85 & 0.75 \\
\hline 0.93 & 1.11 & 0.95 & 1.03 \\
\hline 1.12 & & 0.95 & \\
\hline $1.00 \pm 0.08 *$ & $0.94 \pm 0.20 *$ & $0.95 \pm 0.11 *$ & $0.89 \pm 0.14 *$ \\
\hline \multicolumn{2}{|c|}{$p=0.260$} & \multicolumn{2}{|c|}{$p=0.221$} \\
\hline
\end{tabular}

*Data are mean \pm standard deviation.

Intracranial 3D time-of-flight angiography and contrastenhanced TWIST angiography. All patients with an occluded rCCA showed intracranial (via cross-flow and flow from the 
posterior to the anterior circulation via the anterior communicating artery and the right posterior communicating artery) or extracranial collateral vessels to maintain bilateral cerebral perfusion. Extracranial collateral blood supply was mainly achieved via the thyreocervical trunk, the occipital artery of the right external carotid artery filling the right internal carotid artery (ICA). In all patients with occlusion of the rCCA, the right ICA was patent, but its diameter was reduced in all cases. All children of the control group had a normal cerebral vascular supply.

\section{Discussion}

Sacrifying the CCA after ECMO therapy has often been a matter of discussion in the past and a reason not to perform veno-arterial ECMO therapy. Nowadays, there are two options at the time of decannulation: primary ligation or surgical reconstruction of the CCA. There exists only little information on cerebral perfusion in children after $\mathrm{CDH}$ repair and $\mathrm{rCCA}$ occlusion after ECMO therapy due to primary ligation or secondary occlusion after surgical reconstruction of the vessel.

Our study demonstrated the feasibility of non-contrastenhanced pASL at 3.0 T in 2-year-old children after $\mathrm{CDH}$ repair to quantify cerebral perfusion. Using pASL, we show that 2-year-old patients with rCCA occlusion after neonatal ECMO therapy showed intraindividual side differences between hemispheres, with a reduced cerebral perfusion of the right hemisphere as a common finding. On average, there was a significant difference between the subcortical perfusion of the right and left hemispheres $(p=0.011)$ and a trend towards reduced cortical perfusion of the right hemisphere $(p=0.138)$. In contrast to the ECMO-treated group, the control group without ECMO therapy exhibited nearly identical subcortical and cortical cerebral perfusion of both hemispheres. Although there was no statistically significant difference in the $\mathrm{rCBF}_{\text {right }} / \mathrm{rCBF}_{\text {left }}$ ratio between patients with an occluded rCCA after ECMO therapy and the control group without ECMO therapy, in nearly one-third of the patients with rCCA occlusion (29\%), an intra-individual side difference was found with a cortical and/or subcortical perfusion of the right hemisphere being reduced by more than $20 \%$ compared to the left hemisphere.

However, comparing the absolute values of the $\mathrm{rCBF}$ of the same hemisphere between patients with rCCA occlusion after ECMO therapy and patients of our control group, the mean $\mathrm{rCBF}$ in patients with rCCA occlusion was always higher, with an even significantly higher mean subcortical rCBF of the left hemisphere in patients with rCCA occlusion when compared to that of the control group. Even in those four patients with an occlusion of the rCCA and an intra-individual side difference in $\mathrm{rCBF}$ of more than $20 \%$ between hemispheres, the mean $\mathrm{rCBF}$ of the right hemisphere was quite similar or even higher when compared to that of the control group with a normal cerebral blood supply.

One explanation for $71 \%$ of our patients with rCCA occlusion having no relevant difference in intra-individual perfusion between hemispheres and for the mean $\mathrm{rCBF}$ in patients with rCCA occlusion being similar to or even higher when compared to the control group with a normal cerebral blood supply might be the good collateralization of arterial blood flow in children. In our study cohort, all children with rCCA occlusion showed the presence of sufficient intra- or extracranial collateral vessels to ensure bilateral cerebral perfusion and to prevent hypoperfusion of the ipsilateral hemisphere. As already mentioned, the subcortical rCBF of the left hemisphere in patients with rCCA occlusion was significantly higher when compared with the control group. The higher subcortical rCBF of the left hemisphere in this group reflects the hyperperfusion of the left hemisphere mainly achieved via the left ICA, which is known to be the major collateral vessel in unilateral carotid artery occlusion, to maintain sufficient perfusion of the right hemisphere via collaterals. Previous studies by Neff et al. investigated patients with unilateral ICA occlusion prior to extracranial to intracranial arterial bypass surgery or patients with Moyamoya disease (occlusion/stenosis of one ICA) using 2D cine phase-contrast MRI blood flow quantification. In agreement with our results, they reported significantly higher blood volume flows in the contralateral ICA and the basilar artery when compared to healthy controls, which maintained sufficient blood supply to both hemispheres $(16,17)$. Using Doppler and duplex ultrasound, Matsumoto et al. demonstrated that at the time of rCCA ligation after ECMO therapy, collateral blood flow in newborns was immediately established and further augmented within minutes after ligation (18). Büsing et al. showed that all cases of children with rCCA occlusion after ECMO therapy presented with intra- and extracranial collateral blood supply at the age of 2 years and that unsuccessful rCCA reconstruction was not predictive of a poorer neurological outcome (7). In contrast to this study, Lago et al. identified focal cerebral lesions in $23 \%$ of a cohort of 31 newborns with rCCA ligation after ECMO therapy (19) and McGahren et al. found cerebral abnormalities in $50 \%(6 / 12)$ of children with a permanently ligated rCCA after ECMO therapy (20). In our study, no focal cerebral lesions were found in the group with occlusion of the rCCA. Therefore it is important that future studies with larger patient cohorts systematically investigate the capability of pediatric patients with unilateral occlusion or stenosis of the CCA or the ICA to maintain a sufficient perfusion of the ipsilateral hemisphere via collaterals and to investigate whether or not unilateral vessel occlusion does increase the risk for cerebral lesions compared with children with normal bilateral cerebral blood supply. 
In accordance with our study, former studies evaluating adult patients with arteriosclerosis also demonstrated a side difference regarding $\mathrm{CBF}$ in patients with occlusion or stenosis of the carotid artery using pASL. Bokkers et al. used pASL in patients with symptomatic ICA stenoses. They found significantly reduced CBF in the hemisphere ipsilateral to the stenosis compared with the contralateral hemisphere (21). Using pASL, Hendrikse et al. investigated CBF in the middle cerebral artery territory in patients with ICA occlusion. They observed a significant decrease in $\mathrm{CBF}$ in the hemisphere ipsilateral to the occlusion as compared with the CBF in the contralateral hemisphere and in a healthy control group (22). In contrast to our study, Bokkers and Hendrikse et al. also described significantly reduced $\mathrm{CBF}$ in the hemisphere ipsilateral to the stenosis or occlusion when compared to that of healthy controls $(21,22)$. In contrast to our study, both study groups investigated adult patients. One possible explanation for these contradictory results might be the better capability of children to develop sufficient collateralization even possibly with hyperperfusion of the contralateral hemisphere to maintain a sufficient perfusion of the ipsilateral hemisphere distal to the vessel occlusion.

Because pASL imaging is non-invasive and does not require contrast agent or radioactive tracers, it is an appropriate method for measuring brain perfusion in children $(23,24)$. The number of studies evaluating rCBF by pASL in young children is very limited. Duncan et al. measured $\mathrm{rCBF}$ of different brain regions in 61 healthy children between the ages of 3 and 5 months. This study group reported on statistically significant differences in rCBF between different brain regions and also demonstrated age-related differences in CBF (25). Their results for $\mathrm{rCBF}$ values when extrapolated to 2-year-old children are in good agreement with ours. To our knowledge the present work is the first study evaluating cerebral perfusion in 2-year-old children after $\mathrm{CDH}$ repair. Other studies also demonstrated the feasibility of pASL in pediatric patients. Chen et al. demonstrated the utility of pASL perfusion MRI for characterizing alterations of CBF in children with arterial ischemic stoke and showed a good correlation between pASL perfusion maps and the degree of arterial stenosis (26). Another study reported on the potential of ASL imaging for visualizing decreased perfusion in newborns with perinatal arterial ischemic stroke (27).

Our study has some limitations. Firstly, the study included only a small number of patients, and children with successful rCCA reconstruction after ECMO therapy were not included in the 29 consecutive patients presented. As the present study was designed as a feasibility study, we decided to compare only patients with rCCA occlusion with a control group of patients with $\mathrm{CDH}$ without ECMO therapy and normal cerebral blood supply. Further studies with a larger number of patients need to be carried out to confirm our results and to compare CBF of children with rCCA occlusion with that of children with normal cerebral blood supply after successful reconstruction of the rCAA. Secondly, future studies with a higher number of patients should correlate the pASL data with long-term neurological outcome of patients with $\mathrm{CDH}$. Another limitation is that although larger vessels were excluded, the ROIs still contained smaller vessels. These smaller vessels could have had an impact on the rCBF measurements.

In conclusion, this single-center study showed that noncontrast-enhanced pASL at 3.0 T allows for non-invasive quantitative measurements of brain perfusion in 2-year-old children after $\mathrm{CDH}$ repair. Our study demonstrates that there are intra-individual perfusion differences between cerebral hemispheres in patients with rCCA occlusion after ECMO therapy, with reduced perfusion ipsilateral to the occlusion as a common finding. In nearly one-third of the patients with rCCA occlusion, $\mathrm{rCBF}$ of the right hemisphere was reduced by more than $20 \%$ when compared to the left hemisphere. Although there were intra-individual side differences in $\mathrm{rCBF}$ in patients with rCCA occlusion after ECMO, perfusion of the right hemisphere was sufficient due to collateral blood supply and hyperperfusion of the left hemisphere, with a mean $\mathrm{rCBF}$ that was even higher when compared to the control group.

Based on the results of this study, surgical reconstruction of the rCCA after ECMO therapy remains an issue of discussion and an individual decision of the physicians. Some centers, as we do, still prefer to reconstruct the rCCA to maintain a normal cerebral blood supply with two patent CCAs and to avoid hyperperfusion of the contralateral hemisphere although secondary occlusion after reconstruction can occur.

\section{References}

1 Ackerman KG and Pober BR: Congenital diaphragmatic hernia and pulmonary hypoplasia: New insights from developmental biology and genetics. Am J Med Genet C Semin Med Genet 145C(2): 105-108, 2007.

2 Laudy JA, Van Gucht M, Van Dooren MF, Wladimiroff JW and Tibboel D: Congenital diaphragmatic hernia: An evaluation of the prognostic value of the lung-to-head ratio and other prenatal parameters. Prenat Diagn 23(8): 634-639, 2003.

3 Sola JE, Bronson SN, Cheung MC, Ordonez B, Neville HL and Koniaris LG: Survival disparities in newborns with congenital diaphragmatic hernia: A national perspective. J Pediatr Surg 45(6): 1336-1342, 2010.

4 The Congenital Diaphragmatic Hernia Study Group: Does extracorporeal membrane oxygenation improve survival in neonates with congenital diaphragmatic hernia? J Pediatr Surg 34(5): 720-724, 1999.

5 Danzer E, Gerdes M, Bernbaum J, D'Agostino J, Bebbington MW, Siegle J, Hoffman C, Rintoul NE, Flake AW, Adzick NS and Hedrick HL: Neurodevelopmental outcome of infants with congenital diaphragmatic hernia prospectively enrolled in an interdisciplinary follow-up program. J Pediatr Surg 45(9): 17591766,2010 
6 Jaillard SM, Pierrat V, Dubois A, Truffert P, Lequien P, Wurtz $\mathrm{AJ}$ and Storme L: Outcome at 2 years of infants with congenital diaphragmatic hernia: A population-based study. Ann Thorac Surg 75(1): 250-256, 2003.

7 Buesing KA, Kilian AK, Schaible T, Loff S, Sumargo S and Neff $\mathrm{KW}$ : Extracorporeal membrane oxygenation in infants with congenital diaphragmatic hernia: Follow-up MRI evaluating carotid artery reocclusion and neurologic outcome. Am J Roentgenol 188(6): 1636-1642, 2007.

8 Desai SA, Stanley C, Gringlas M, Merton DA, Wolfson PJ, Needleman L, Graziani LJ and Baumgart S: Five-year followup of neonates with reconstructed right common carotid arteries after extracorporeal membrane oxygenation. J Pediatr 134(4): 428-433, 1999.

9 Wang J and Licht DJ: Pediatric perfusion MR imaging using arterial spin labeling. Neuroimag Clinics North Am 16(1): 149167,2006

10 Alsop DC, Detre JA, Golay X, Gunther M, Hendrikse J, Hernandez-Garcia L, Lu H, Macintosh BJ, Parkes LM, Smits M, van Osch MJ, Wang DJ, Wong EC and Zaharchuk G: Recommended implementation of arterial spin-labeled perfusion mri for clinical applications: A consensus of the ISMRM Perfusion Study Group and the European Consortium for ASL in Dementia. Magn Reson Med 73(1): 102-116, 2015.

11 De Vis JB, Petersen ET, de Vries LS, Groenendaal F, Kersbergen KJ, Alderliesten T, Hendrikse $\mathrm{J}$ and Benders MJ: Regional changes in brain perfusion during brain maturation measured non-invasively with arterial spin labeling MRI in neonates. Eur J Radiol 82(3): 538-543, 2013.

12 Reiss I, Schaible T, van den Hout L, Capolupo I, Allegaert K, van Heijst A, Gorett Silva M, Greenough A and Tibboel D: Standardized postnatal management of infants with congenital diaphragmatic hernia in Europe: The CDH Euro Consortium Consensus. Neonatology 98(4): 354-364, 2010.

13 Waag KL, Loff S, Zahn K, Ali M, Hien S, Kratz M, Neff W, Schaffelder R and Schaible T: Congenital diaphragmatic hernia: A modern day approach. Semin Pediatr Surg 17(4): 244-254, 2008.

14 Luh WM, Wong EC, Bandettini PA and Hyde JS: Quipss ii with thin-slice til periodic saturation: A method for improving accuracy of quantitative perfusion imaging using pulsed arterial spin labeling. Magn Reson Med 41(6): 1246-1254, 1999.

15 Lim RP, Shapiro M, Wang EY, Law M, Babb JS, Rueff LE, Jacob JS, Kim S, Carson RH, Mulholland TP, Laub G and Hecht EM: 3d time-resolved $\mathrm{mr}$ angiography (mra) of the carotid arteries with time-resolved imaging with stochastic trajectories: Comparison with 3D contrast-enhanced bolus-chase MRA and 3D time-of-flight MRA. Am J Neuroradiol 29(10): 1847-1854, 2008.

16 Neff KW, Horn P, Dinter D, Vajkoczy P, Schmiedek P and Duber $\mathrm{C}$ : Extracranial-intracranial arterial bypass surgery improves total brain blood supply in selected symptomatic patients with unilateral internal carotid artery occlusion and insufficient collateralization. Neuroradiology 46(9): 730-737, 2004.

17 Neff KW, Horn P, Schmiedek P, Duber C and Dinter DJ: 2D Cine phase-contrast mri for volume flow evaluation of the brainsupplying circulation in Moyamoya disease. Am J Roentgenol 187(1): W107-115, 2006.
18 Matsumoto JS, Babcock DS, Brody AS, Weiss RG, Ryckman FG and Hiyama D: Right common carotid artery ligation for extracorporeal membrane oxygenation: Cerebral blood flow velocity measurement with Doppler duplex US. Radiology 175(3): 757-760, 1990.

19 Lago P, Rebsamen S, Clancy RR, Pinto-Martin J, Kessler A, Zimmerman R, Schmelling D, Bernbaum J, Gerdes M, D'Agostino JA and Baumgart S: MRI, MRA, and neurodevelopmental outcome following neonatal ECMO. Pediatr Neurol 12(4): 294-304, 1995.

20 McGahren ED, Mallik K and Rodgers BM: Neurological outcome is diminished in survivors of congenital diaphragmatic hernia requiring extracorporeal membrane oxygenation. J Pediatr Surg 32(8): 1216-1220, 1997.

21 Bokkers RP, van Laar PJ, van de Ven KC, Kapelle LJ, Klijn CJ and Hendrikse $\mathrm{J}$ : Arterial spin-labeling MR imaging measurements of timing parameters in patients with a carotid artery occlusion. Am J Neuroradiol 29(9): 1698-1703, 2008.

22 Hendrikse J, van Osch MJ, Rutgers DR, Bakker CJ, Kappelle LJ, Golay X and van der Grond J: Internal carotid artery occlusion assessed at pulsed arterial spin-labeling perfusion MR imaging at multiple delay times. Radiology 233(3): 899-904, 2004.

23 Wang J, Licht DJ, Jahng GH, Liu CS, Rubin JT, Haselgrove J, Zimmerman RA and Detre JA: Pediatric perfusion imaging using pulsed arterial spin labeling. J Magn Reson Imaging 18(4): 404413, 2003.

24 Bokkers RP, Bremmer JP, van Berckel BN, Lammertsma AA, Hendrikse J, Pluim JP, Kappelle LJ, Boellaard R and Klijn CJ: Arterial spin labeling perfusion mri at multiple delay times: A correlative study with $\mathrm{H}(2)(15) \mathrm{O}$ positron-emission tomography in patients with symptomatic carotid artery occlusion. J Cereb Blood Flow Metab 30(1): 222-229, 2010.

25 Duncan AF, Caprihan A, Montague EQ, Lowe J, Schrader R and Phillips JP: Regional cerebral blood flow in children from 3 to 5 months of age. Am J Neuroradiol 35(3): 593-598, 2014.

26 Chen J, Licht DJ, Smith SE, Agner SC, Mason S, Wang S, Silvestre DW, Detre JA, Zimmerman RA, Ichord RN and Wang $\mathrm{J}$ : Arterial spin labeling perfusion MRI in pediatric arterial ischemic stroke: Initial experiences. J Magn Reson Imaging 29(2): 282-290, 2009.

27 De Vis JB, Petersen ET, Kersbergen KJ, Alderliesten T, de Vries LS, van Bel F, Groenendaal F, Lemmers PM, Hendrikse J and Benders MJ: Evaluation of perinatal arterial ischemic stroke using noninvasive arterial spin labeling perfusion MRI. Pediatr Res 74(3): 307-313, 2013. 